\title{
Risk factors for discontinuation of exclusive breast feeding within 1-month: A retrospective cohort study
}

\author{
Shun Yasuda ( $\square$ room335@fmu.ac.jp ) \\ Iwase General Hospital https://orcid.org/0000-0002-6520-902X \\ Toma Fukuda \\ Iwase General Hospital \\ Naoya Toba \\ Iwase General Hospital \\ Norihito Kamo \\ Iwase General Hospital \\ Karin Imaizumi \\ Iwase General Hospital \\ Midori Yokochi \\ Iwase General Hospital
}

Tomoko Okawara

Iwase General Hospital

Seiko Takano

Iwase General Hospital

Hideko Yoshida

Iwase General Hospital

Nobuko Kobayashi

Iwase General Hospital

Shingo Kudo

Iwase General Hospital

Kyohei Miyazaki

Iwase General Hospital

Mamiko Hosoya

Iwase General Hospital

Kenichi Sato

Iwase General Hospital

Kei Takano

Iwase General Hospital

Aya Kanno 
Fukushima Medical University

\section{Tsuyoshi Murata}

Fukushima Medical University

\section{Hyo Kyozuka}

Fukushima Medical University

\section{Akiko Yamaguchi}

Fukushima Medical University

\section{Fumihiro Ito}

Iwase General Hospital

\section{Shinichiro Oda}

Iwase General Hospital

Nobuo Momoi

Fukushima Medical University

\section{Mitsuaki Hosoya}

Fukushima Medical University

\section{Keiya Fujimori}

Fukushima Medical University

\section{Research}

Keywords: Breast feeding, smoking, Japan, postpartum period

Posted Date: October 2nd, 2020

DOI: https://doi.org/10.21203/rs.3.rs-31236/v2

License: (c) (1) This work is licensed under a Creative Commons Attribution 4.0 International License.

Read Full License 


\section{Abstract}

Background: While breastfeeding provides both optimal nutrition for infants and health benefits for the mother, many women either do not breastfeed or terminate breastfeeding earlier than recommended. The aim of this analysis was to identify factors associated with early discontinuation of breastfeeding in Japanese women.

Methods: This study utilized medical records from the Iwase General Hospital in Fukushima Prefecture, Japan to assess cessation of breastfeeding by the 1-month postpartum appointment. Demographic, medical, and family predictors among 734 women who had initiated breastfeeding during their delivery hospital stay were examined, and multiple logistic regression was used to determine significant predictors of early cessation of exclusive breastfeeding.

Results: In a bivariate analysis, it was observed that women who were primipara, unmarried, smoked before pregnancy, were exposed to secondhand smoke, employed, and/or had asthma were more likely to discontinue exclusive breastfeeding. Infant factors associated with discontinuation were lower birthweight, earlier gestational age, neonatal intensive care unit admission, treatment for jaundice, or lower weight gain. In multivariable analysis, primiparity, smoking before pregnancy, maternal employment and neonatal jaundice therapy remained associated with discontinuation of breastfeeding.

Conclusions: Several maternal factors are associated with discontinuation of exclusive breastfeeding. In particular, women who smoke before pregnancy may need to be targeted for additional support for breastfeeding.

\section{Background}

Breastfeeding is generally considered to provide optimal nutrition for babies. Infants who are breastfed have lower rates of infection, better cognition, and a reduced risk of obesity; although the extent to which this relation is causal, especially in the developed countries, is not completely clear. Breastfeeding also has important benefits for maternal health, including reduced risk of depression [1] and reduced cardiovascular and metabolic risk later in life [2]. As these benefits are most strongly seen in women and infants who breastfeed the longest $[3,4]$, it follows that early cessation reduces the possible improvements. In Japan, over $95 \%$ of women attempt to breastfeed, but this declines dramatically in the first month, with $50 \%$ or less breastfeeding at 1 -month postpartum [5]. Breastfeeding is strongly encouraged, and clinicians work together to establish breastfeeding before a woman leaves the hospital post-delivery. However, many women still discontinue breastfeeding shortly after, even within the first month, well before supplementation is optimal.

Many women experience difficulties breastfeeding, including physical symptoms and logistical barriers such as returning to work [6, 7]. In addition, breastfeeding is a dynamic interaction, and women who believe that they are not providing sufficient quantities of milk, or that their infant is not gaining a 
sufficient amount of weight are more likely to supplement or discontinue breastfeeding altogether [6, 810].

The goal of this analysis was to identify factors associated with early discontinuation of breastfeeding, with a particular focus on factors relevant to Japanese women - where breastfeeding is highly socially accepted, but exclusive breastfeeding for the recommended time is less common [11]. Breastfeeding practices are not universal, and previous studies on predictors of breastfeeding in Japan found mixed associations with age and education- factors that are strongly associated with breastfeeding in other countries [5]. Previous studies have indicated that living with the baby's father promotes breastfeeding, while living with a grandparent does not [12]. The information from this study will assist with the development of clinical practice and other programs that may mitigate the risk of early discontinuation of breastfeeding.

\section{Methods}

\section{Study setting and population}

This study was conducted at Iwase General Hospital, the only general public hospital serving the Fukushima Prefecture in Japan. The hospital has a small neonatal intensive care unit ( 3 beds) that can treat infants born at 34 weeks' gestation or later, and averages 50 deliveries per month. In Japan, $99 \%$ of women deliver in a hospital or clinic, and the average hospital stay associated with delivery is 5 days. The hospital follows the World Health Organization's guidelines for establishing breastfeeding, including facilitating skin-to-skin contact, allowing rooming-in, and not providing breastmilk substitutes [13].

\section{Study design}

This study was a retrospective cohort, created by abstracting electronic medical records from a single hospital. Medical records from women delivering a singleton livebirth between March 2017 and August 2019 were included in this analysis; a total of 1350 women met this standard. Women were excluded if they delivered preterm ( $<37$ weeks' gestation, $n=77$ ), had an infant with birthweight $<2500 \mathrm{~g}$ or $<1.5 \mathrm{SD}$ the mean for gestational age $(n=148)$ and remained in the neonatal intensive care unit $(N I C U)$ at time of mother's discharge $(n=104)$, or had any serious maternal complications (hemolysis, elevated liver enzymes, low platelet count [HELLP], $n=2$; severe hemorrhage, $n=2$ ). This left a total of 1104 women with no obvious contraindication to breastfeeding at the time of birth; of these, 734 (66.4\%) had established breastfeeding by the time the mother left the hospital. All women returned for a 1-month infant/maternal visit.

\section{Outcome}

The outcome was discontinuation of exclusive breastfeeding by the 1-month postpartum visit, defined as complete cessation of breastfeeding or supplementation with formula. This was based on a standard questionnaire and clinician interview about the child's nutrition; the responses were (1) complete 
breastfeeding, (2) supplemental formula (at the time of the 1-month checkup, the mother was feeding the infant with formula, even if only a little, in addition to breast milk), and (3) formula (at the time of the 1month checkup, the infant was being fed exclusively with formula).

\section{Predictors}

Demographic, medical, behavioral, and family factors for both the mother and infant were examined as possible predictors of breastfeeding discontinuation. All factors were abstracted from the women's medical records. The demographic factors examined were age at birth, marital status, and employment status (any job at time of birth). The maternal medical factors examined were parity, underlying diseases (physical condition, such as thyroid disease or diabetes, or mental condition, such as anxiety or mood disorder), allergies, pre-pregnancy weight/body mass index (BMI), type of delivery (cesarean or vaginal), use of infertility treatment (assisted reproductive technology), hemorrhage, and mastitis. The infant medical factors were birthweight, gestational age, sex, Apgar score, umbilical artery $\mathrm{pH}$, admission of infant to NICU after day-1, jaundice, and weight gain per day. Behavioral factors examined were prepregnancy smoking, alcohol consumption (any/none), dietary supplements, and preference for exclusive breastfeeding, asked before birth. No women admitted to active or passive smoking during pregnancy. Family factors examined were husband/partner present, family members living at the same house, housing (single-family/multiple dwelling), and returned to parents' house to give birth.

\section{Analysis}

Women who were breastfeeding exclusively at 1-month were compared to those supplementing with formula or using it exclusively. Chi-square tests (Fisher's exact test when cell size was $<5$ ) were used to analyze categorical/dichotomous variables, and t-tests were used for continuous variables. Variables that showed a statistically significant difference $(p<0.05)$ in bivariate analysis were included in a logistic model to identify the strongest predictors. All analyses were conducted using SPSS v. 26.

This study was approved by the Ethics Committee of Iwase General Hospital (\#191102).

\section{Results}

The study population is described in Table 1. The total smoking rate before pregnancy was $17.3 \%$, and the smoking rate among those aged $<30$ years old was statistically higher compared to those aged $\geq 30$ years old $(22.5 \%$ vs. $13.2 \%, p<0.01)$. Women were less likely to continue breastfeeding if they were unmarried $(7.2 \%$ unmarried among those who supplemented with formula vs. $2.9 \%$ unmarried among those who continued exclusive breastfeeding, $p=0.01)$, smoked before pregnancy $(26.8 \%$ vs. $13.5 \%, p<$ $0.01)$, had a partner who smoked ( $34.4 \%$ vs. $23.1 \%, p<0.01)$, were employed $(70 \%$ vs. $61.8 \%, p=0.04)$, or had asthma ( $13.2 \%$ vs. $6.7 \%, p<0.01)$. Infant factors associated with discontinuation were lower birthweight (mean birthweight $3017 \mathrm{~g}$ in those who were supplemented vs. $3105 \mathrm{~g}$ in those exclusively breastfed), earlier gestational age (39w4d vs. $39 w 5 d, p<0.01)$, NICU admission $(12.9 \%$ vs. $7.6 \%, p<$ 
$0.01)$, neonatal jaundice treatment $(12.0 \%$ vs. $5.5 \%, \mathrm{p}<0.01)$, and lower weight gain $(42.4$ vs. $45.7, \mathrm{p}<$ $0.01)$.

In multivariable analysis (Table 2), primiparity (adjusted odds ratio (aOR), 1.69; 95\% confidence interval $(\mathrm{Cl}), 1.21-2.36)$, active smoking before pregnancy (aOR, 2.00; 95\% $\mathrm{Cl}, 1.31-3.02)$, maternal employment (aOR, 1.50; 95\% Cl, 1.05-2.15), and neonatal jaundice treatment (aOR, 2.15; 95\% $\mathrm{Cl}, 1.20-2.36$ ) remained associated with discontinuation of breastfeeding.

\section{Discussion}

Even if breastfeeding was well established when mothers were discharged from hospital after giving birth, primiparity, employment, smoking before pregnancy, and infant jaundice requiring phototherapy were associated with an increased risk of discontinuation of breastfeeding. The associations with primiparity and employment were already known. Previous studies in Japan have found that employed mothers are less likely to breastfeed exclusively (although breastfeeding is more common in those with extended leave than those who were unemployed), while previous studies of parity have had mixed results [5].

Mothers who smoked before becoming pregnant were also at high risk of discontinuing breastfeeding, a factor which has been identified previously. In this study, although all pregnant women who smoked before pregnancy were strictly discouraged from smoking, and no women admitted to either active or passive smoking during pregnancy, smoking before pregnancy was identified to be an independent risk factor for discontinuation of breastfeeding. (It is also possible that some women continued smoking through pregnancy, as the self-reports were not confirmed via biomarker, for instance.) This is important

as it demonstrates that quitting smoking after pregnancy begins may be too late to affect breastfeeding continuation.

Furthermore, smoking before pregnancy was more common in younger women ( $<30$ years old) than older ( $\geq 30$ years old), which is concerning for regional public health as it implies that smoking may be increasing among women of reproductive age. Fukushima Prefecture has one of the highest smoking rates in Japan. According to the Cancer Registry and Statistics of the Cancer Information Service of the National Cancer Center, Japan, $10.7 \%$ of women aged $\geq 20$ years are smokers, ranking it as the eighth worst prefecture in Japan (as of 2016). In fact, the pre-pregnancy smoking rate of women who deliver in our hospital is even higher at $23 \%$.

The reason that this group is at higher risk of supplementing with formula after leaving hospital is unknown. There are no obvious factors that would cause smoking before pregnancy to inhibit the secretion of breastmilk after delivery, but several studies confirm this association [14]. Many smokers who quit during pregnancy relapse, and there is some evidence that milk volume may be reduced and infants' response to breastmilk affected by active smoking [15]. Some women may also worry that chemicals may be passed to the baby through breastmilk or may find the recommendations to limit breastfeeding to several hours after smoking difficult to follow. Among women who do not relapse, it 
seems likely that there is no direct association, but instead correlations with other lifestyle or psychosocial factors. Indeed, studies in smokers who quit during pregnancy found that smoking did not affect breastfeeding decisions for the majority, although some reduced smoking during breastfeeding [16]. Smokers did not differ from non-smokers in their reported reasons for ceasing breastfeeding [17]. However, one previous study of smoking prior to delivery found that women who intended to breastfeed were more likely to quit smoking during pregnancy [18], so reverse causation is a possibility: women who intend to revert to smoking may choose not to breastfeed. Exposure to secondhand smoke, more common among women who smoke, has also been associated with breastfeeding cessation $[19,20]$.

Novel solutions may be needed to address these issues; smoking cessation through pediatric practices, rather than limited to primary or gynecologic care, shows promise [21]. Several evidence-based programs to improve cessation during pregnancy and prevent relapse have been shown to be effective [22], although not all have been tested in a Japanese population and relapse rates are still quite high [23]. A mix of behavioral and incentive strategies may be most effective [24]. It is the authors' experience that discussing the risk of Sudden Infant Death Syndrome and other risks to the infant's health are effective in encouraging women to quit smoking.

Neonatal jaundice treatment was associated with an increased likelihood of discontinuation of breastfeeding. A previous Canadian study found no differences in discontinuation between infants hospitalized for hyperbilirubinemia and those who were not [25], while an Italian study similarly failed to find jaundice predictive [26]. However, as "breastfeeding jaundice" is a common finding shortly after birth, and may be alarming to parents, it seems reasonable that its presence might contribute to discontinuation.

Several factors that are generally considered to be associated with breastfeeding cessation were not predictive in this analysis. After adjustment, associations with lower infant weight gain were too small to be considered clinically relevant. Previous studies have identified pre-pregnancy BMI and pregnancy weight gain as important predictors (sometimes interacting with smoking) [27], which was not the case in the current analysis. In this study, differences in birthweight for gestational age (a factor shown to be associated with discontinuation in other studies [28]) by breastfeeding status were identified, but these differences were not considered to be clinically significant, and consequently were not included in further analysis.

The strengths of the study include the consideration of medical, individual, and family factors, as well as the extended follow-up. Furthermore, as none of the women reported smoking during pregnancy, the study isolates the effect of pre-pregnancy smoking. The limitations of the current study include the lack of information on maternal mental health during pregnancy or postpartum (unlike now, at the time of the study, depression was not usually included in the medical record), or difficulties in breastfeeding beyond mastitis. In addition, no information was available on attitudes and intentions around breastfeeding [29], pacifier use (not recommended by the hospital) [30], or previous breastfeeding among multiparous 
women. Moreover, other studies have explored various factors that were unable to be assessed here. For instance, formula supplementation in the hospital has been shown to be associated with an increased likelihood of cessation [31]. Intention to breastfeed and length of intended time are also strongly associated [32, 33]. In addition, perceived insufficient milk supply, breast problems, and tiredness were all associated with stopping breastfeeding before 3 months in a cohort of women who intended to continue breastfeeding [31]. Lack of confidence in breastfeeding and belief that the baby prefers formula were associated with stopping breastfeeding in the first 2 weeks in a cohort of lower-income women in Connecticut (USA), as were young maternal age and lower education [33].

\section{Conclusion}

Although the mechanism is unclear, this epidemiological study suggests that smoking before pregnancy is a risk factor for the later non-continuation of breastfeeding. If this is the case, preconception education may be required to encourage women of childbearing age to quit smoking before they begin planning pregnancy. If these findings go some way to enable infants to breastfeed, then this study will be extremely important. Further studies on smoking cessation as a component of preconception care are also planned.

\section{List Of Abbreviations}

BMI, body mass index; NICU, neonatal intensive care unit; aOR, adjusted odds ratio; $\mathrm{Cl}$, confidence interval

\section{Declarations}

Ethical approval and consent to participate: The study was approved by the Ethics Committee of Iwase Hospital (\#191102).

Consent for publication: Not applicable

Data availability: The datasets generated and analyzed in the current study are not publicly available due to patient confidentiality concerns but are available from the corresponding author on reasonable request.

Competing interest: The authors declare that they have no competing interests.

Funding: None.

\section{Authors' contributions:}

Conception and design of study: Shun Yasuda, Nobuko Kobayashi, Shinichiro Oda

Acquisition of data: Toma Fukuda, Naoya Toba, Norihito Kamo, Midori Yokochi, Tomoko Okawara, Seiko Takano, and Hideko Yosihida 
Analysis and/or interpretation of data: Shun Yasuda, Toma Fukuda, Naoya Toba, Norihito Kamo Shingo Kudo, Kyohei Miyazaki, Mamiko Hosoya, Kenichi Sato Kei Takano, and Shinichiro Oda

Drafting the manuscript: Shun Yasuda, Toma Fukuda, Naoya Toba, and Shinichiro Oda

Revising the manuscript critically for important intellectual content: Shun Yasuda, Toma Fukuda, Naoya Toba, Shinichiro Oda, Karin Imaizumi, Aya Kanno, Tsuyoshi Murata, Hyo Kyozuka, Akiko Yamaguchi, Shinichiro Oda, Fumihiro Ito, Nobuo Momoi, Mitsuaki Hosoya, and Keiya Fujimori

Acknowledgments: We would like to acknowledge the support of the Department of Obstetrics and Gynecology of Fukushima Medical University. Editorial support, in the form of medical writing, assembling tables and creating high-resolution images based on the authors' detailed directions, collating author comments, copyediting, fact checking, and referencing, was provided by Editage.

\section{References}

1. Kendall-Tackett $\mathrm{K}$. The new paradigm for depression in new mothers: current findings on maternal depression, breastfeeding and resiliency across the lifespan. Breastfeed Rev. 2015;23:7-10.

2. Rameez RM, Sadana D, Kaur S, Ahmed T, Patel J, Khan MS, et al. Association of maternal lactation with diabetes and hypertension: a systematic review and meta-analysis. JAMA Netw Open. 2019;2:e1913401.

3. Chowdhury R, Sinha B, Sankar MJ, Taneja S, Bhandari N, Rollins N, et al. Breastfeeding and maternal health outcomes: a systematic review and meta-analysis. Acta paediatrica (Oslo, Norway : 1992). 2015;104:96-113.

4. Kramer MS, Kakuma R. Optimal duration of exclusive breastfeeding. Cochrane Database Syst Rev. 2012;2012:Cd003517.

5. Inoue M, Binns CW, Otsuka K, Jimba M, Matsubara M. Infant feeding practices and breastfeeding duration in Japan: a review. Int Breastfeed J. 2012;7:15.

6. Gianni ML, Bettinelli ME, Manfra P, Sorrentino G, Bezze E, Plevani L, et al. Breastfeeding difficulties and risk for early breastfeeding cessation. Nutrients. 2019;11:2266.

7. Ahmadi M, Moosavi SM. Evaluation of occupational factors on continuation of breastfeeding and formula initiation in employed mothers. Glob J Health Sci. 2013;5:166-71.

8. Roostaee F, Tabatabaei SM, Zaboli M, Keykhaie R, Sharifi-Rad J, Shahrak P, et al. Breast-feeding continuation in south-eastern of Iran: the associated factors. Med Arch. 2015;69:98-102.

9. Kent JC, Hepworth AR, Sherriff JL, Cox DB, Mitoulas LR, Hartmann PE. Longitudinal changes in breastfeeding patterns from 1 to 6 months of lactation. Breastfeed Med. 2013;8:401-7. 
10. Kramer MS, Moodie EE, Dahhou M, Platt RW. Breastfeeding and infant size: evidence of reverse causality. Am J Epidemiol. 2011;173:978-83.

11. Haku M. Breastfeeding: Factors associated with the continuation of breastfeeding, the current situation in Japan, and recommendations for further research. J Med Invest. 2007;54:224-34.

12. Kaneko A, Kaneita Y, Yokoyama E, Miyake T, Harano S, Suzuki K, et al. Factors associated with exclusive breast-feeding in Japan: for activities to support child-rearing with breast-feeding. J Epidemiol. 2006;16:57-63.

13. World Health Organization. Ten steps to successful breastfeeding [https://www.who.int/nutrition/bfhi/ten-steps/en/]. Accessed 2019 December 19.

14. Lechosa Muniz C, Paz-Zulueta M, Del Rio EC, Sota SM, Saez de Adana M, Perez MM, et al. Impact of maternal smoking on the onset of breastfeeding versus formula feeding: a cross-sectional study. Int $J$ Environ Res Public Health. 2019;16:4888.

15. Napierala M, Mazela J, Merritt TA, Florek E. Tobacco smoking and breastfeeding: Effect on the lactation process, breast milk composition and infant development. A critical review. Environ Res. 2016;151:321-38.

16. Joseph HM, Emery RL, Bogen DL, Levine MD. The influence of smoking on breast feeding among women who quit smoking during pregnancy. Nicotine Tob Res. 2017;19:652-5.

17. Weiser TM, Lin M, Garikapaty V, Feyerharm RW, Bensyl DM, Zhu BP. Association of maternal smoking status with breastfeeding practices: Missouri, 2005. Pediatrics. 2009;124:1603-10.

18. Carswell AL, Ward KD, Vander Weg MW, Scarinci IC, Girsch L, Read M, et al. Prospective associations of breastfeeding and smoking cessation among low-income pregnant women. Matern Child Nutr. 2018;14:e12622.

19. Lok KYW, Wang MP, Chan VHS, Tarrant M. Effect of secondary cigarette smoke from household members on breastfeeding duration: a prospective cohort study. Breastfeed Med. 2018;13:412-7.

20. Suzuki D, Wariki WMV, Suto M, Yamaji N, Takemoto Y, Rahman M, et al. Secondhand smoke exposure during pregnancy and mothers' subsequent breastfeeding outcomes: a systematic review and meta-analysis. Sci Rep. 2019;9:8535.

21. Drehmer JE, Ossip DJ, Nabi-Burza E, Hipple Walters B, Gorzkowski JA, Winickoff JP. Pediatric office delivery of smoking cessation assistance for breast-feeding mothers. Nicotine Tob Res. 2018;22:346-53.

22. Chamberlain C, O'Mara-Eves A, Porter J, Coleman T, Perlen SM, Thomas J, et al. Psychosocial interventions for supporting women to stop smoking in pregnancy. Cochrane Database Syst Rev. 
23. Jones M, Lewis S, Parrott S, Wormall S, Coleman T. Re-starting smoking in the postpartum period after receiving a smoking cessation intervention: a systematic review. Addiction. 2016;111:981-90.

24. Su A, Buttenheim AM. Maintenance of smoking cessation in the postpartum period: which interventions work best in the long-term? Matern Child Health J. 2014;18:714-28.

25. Pound CM, Gaboury I. The impact of jaundice in newborn infants on the length of breastfeeding. Paediatr Child Health. 2009;14:445-9.

26. Govoni L, Ricchi A, Molinazzi MT, Galli MC, Putignano A, Artioli G, et al. Breastfeeding pathologies: analysis of prevalence, risk and protective factors. Acta Biomed. 2019;90:56-62.

27. Vurbic D, Higgins ST, McDonough SR, Skelly JM, Bernstein IM. Maternal body mass index moderates the influence of smoking cessation on breast feeding. Nicotine Tob Res. 2014;16:527-35.

28. Quinlivan J, Kua S, Gibson R, McPhee A, Makrides MM. Can we identify women who initiate and then prematurely cease breastfeeding? An Australian multicentre cohort study. Int Breastfeed J. 2015;10:16.

29. Robert E, Coppieters Y, Swennen B, Dramaix M. Breastfeeding duration: A survival analysis-data from a regional immunization survey. Biomed Res Int. 2014;2014:529790.

30. Buccini G, Perez-Escamilla R, D'Aquino Benicio MH, Justo Giugliani ER, Isoyama Venancio S. Exclusive breastfeeding changes in Brazil attributable to pacifier use. PLoS One. 2018;13:e0208261.

31. Chan SM, Nelson EA, Leung SS, Li CY. Breastfeeding failure in a longitudinal post-partum maternal nutrition study in Hong Kong. J Paediatr Child Health. 2000;36:466-71.

32. Blyth RJ, Creedy DK, Dennis CL, Moyle W, Pratt J, De Vries SM, et al. Breastfeeding duration in an Australian population: The influence of modifiable antenatal factors. J Hum Lact. 2004;20:30-8.

33. Ertem IO, Votto N, Leventhal JM. The timing and predictors of the early termination of breastfeeding. Pediatrics. 2001;107:543-8.

\section{Tables}


Table 1. Maternal and infant factors associated with discontinuation of exclusive breastfeeding in Japanese women $(\mathrm{n}=734)$

Predictor ( $\mathrm{n}$ with data)

Formula supplementation

n (\%) or mean

\section{Demographics}

Primiparity

$120(57.4 \%)$

Age $(n=734)$

Age $<30$ years

Unmarried marital status

Employed

\section{Health behavior}

Active smoking before pregnancy (n

$=734)$

Passive smoking before pregnancy

$(\mathrm{n}=654)$

Alcohol use before pregnancy

$(\mathrm{n}=732)$
(SD)

$29.6(6.0)$

$56(26.8 \%)$

$145(70.0 \%)$

$15(7.2 \%)$

$15(2.9 \%)$

0.01

$30.0(4.7)$

$230(71.0 \%)$

0.77

0.01

$94(29.0 \%)$

breastfeeding value

n (\%) or mean

(SD)

$229(43.6 \%)$

$<$

0.31

$322(61.8 \%)$

0.04

$72(34.4 \%)$

$103(23.1 \%)$

$<$

0.01

$45(21.5 \%)$

$90(17.2 \%)$

0.17

Dietary supplement use $(n=732)$

$70(33.5 \%)$

$142(27.1 \%)$

0.09 
Pre-pregnancy weight (kg)

Pre-pregnancy BMI $(\mathrm{n}=685)$

Weight gain during pregnancy

$(\mathrm{n}=679)$

\section{Medical}

Food allergy

$(\mathrm{n}=734)$

Drug allergy

$(\mathrm{n}=734)$

Bronchial asthma

$(\mathrm{n}=701)$

Underlying maternal disease

Preference for exclusive

breastfeeding if possible

$(\mathrm{n}=429)$

Mastitis

Infertility treatment $(\mathrm{n}=734)$
$53.7(9.2)$

$53.8(9.3)$

$21.7(3.7)$

$21.5(3.5)$

0.53

$10.2(4.2)$

$9.8(4.3)$

0.29

\section{Family and housing}

Returned to parents' house to give 
birth $(\mathrm{n}=690)$

Post-discharge living environment (n

$$
\text { =167) }
$$

nuclear family

$19(34.5 \%)$

$33(29.5 \%)$

living with own family

$29(52.7 \%)$

$59(52.7 \%)$

living with husband/partner's family

$7(12.7 \%)$

$20(17.9 \%)$

Detached house

$37(74.0 \%)$

$91(84.3 \%)$

0.13

\section{Infant factors}

Vaginal delivery

$(\mathrm{n}=734)$

Birthweight

3017 (285)

3105 (294)

$(\mathrm{n}=734)$

0.01

Gestational age

$(\mathrm{n}=734)$

0.01

Male infant

$115(55.0 \%)$

$248(47.2 \%)$

0.06

$(\mathrm{n}=734)$

5-minute Apgar score $(\mathrm{n}=734)$

$8.9(0.3)$

$8.9(0.4)$

0.81

NICU admission

$27(12.9 \%)$

$40(7.6 \%)$

0.11

$(\mathrm{n}=734)$

Neonatal jaundice treatment $(\mathrm{n}=$

$25(12.0 \%)$

$29(5.5 \%)$

$<$ 734) 
$(\mathrm{n}=734)$

Weight gain at 1-month checkup

*Chi-square test for categorical/dichotomous variables; t-test for continuous variables. If $\mathrm{N}$ is not specified, data were complete. $\mathrm{SD}$, standard deviation

Table 2. Multivariable predictors of discontinuation of exclusive breastfeeding among Japanese women $(\mathrm{n}=734)^{\mathrm{a}}$

\begin{tabular}{lccc}
\hline & aOR & $95 \%$ CI & p-value \\
Primiparity & 1.688 & $1.208-2.359$ & $<0.01$ \\
\hline Employed & 1.502 & $1.049-2.148$ & 0.026 \\
\hline Married & 1.788 & $0.822-3.889$ & 0.142 \\
\hline Active smoking before pregnancy & & & \\
\hline & 1.995 & $1.317-3.021$ & $<0.01$ \\
\hline Neonatal jaundice treatment & & & $<0.01$ \\
\hline
\end{tabular}

${ }^{a}$ All variables statistically significant $(p<0.05)$ in bivariate analysis were included in the multivariable model. aOR, adjusted odds ratio; CI, confidence interval. 